\title{
Sasakian manifolds with vanishing C-Bochner curvature tensor
}

\author{
E-S. Choi (Kyungsan)*, U-H. Ki (Taegu)* and K. Takano (Nagano)
}

\section{Introduction}

As a complex analogue to the Weyl conformal curvature tensor, Bochner and Yano [1], [15] (See also, Tachibana [13]) introduced a Bochner curvature tensor in a Kählerian manifold. Many subjects for vanishing Bochner curvature tensors with constant scalar curvature have been studied by Ki and Kim [6], Kubo [8], Matsumoto [9], Matsumoto and Tanno [11], Yano and Ishihara [16] and so on. One of those, done by Ki and Kim, asserts that the following theorem:

Theorem A ([6]) Let $M$ be a Kählerian manifold with vanishing Bochner curvature tensor. Then the scalar curvature is constant if and only if $\operatorname{Tr} \operatorname{Ric}^{(m)}$ is constant for a positive integer $m(\geq 2)$.

In a Sasakian manifold, a C-Bochner curvature tensor is constructed from the Bochner curvature tensor in a Kählerian manifold by the fibering of Boothby-Wang. Recently, the Sasakian manifold with vanishing C-Bochner curvature tensor and the constant scalar curvature is studied, and in [12], the following theorem was proved

THEOREM B Let $M^{n}(n \geq 5)$ be a Sasakian manifold with constant scalar curvature whose C-Bochner curvature tensor vanishes. If the Ricci tensor is positive semi-definite, then $M$ is a space of constant $\phi$-holomorphic sectional curvature.

Also, when $M$ is compact, the following theorems were proved:

Theorem C ([4]) Let $M^{n}(n \geq 5)$ be a compact Sasakian manifold with vanishing $C$ Bochner curvature tensor. If the length of the Ricci tensor is constant and the length of the $\eta$-Einstein tensor is less than $\frac{\sqrt{2}(R-n+1)}{\sqrt{(n-1)(n-3)}}$, then $M$ is a space of constant $\phi$-holomorphic sectional curvature.

Theorem D ([10]) Let $M^{n}(n \geq 5)$ be a compact Sasakian manifold with vanishing $C$ Bochner curvature tensor and the constant scalar curvature. If the smallest Ricci curvature greater than -2, then $M$ is a space of constant $\phi$-holomorphic sectional curvature.

We shall prove Theorem A as a Sasakian analogue in $\S 3$. Moreover in $\S 4$ we shall discuss when the smallest Ricci curvature is greater than or equal to -2 in a Sasakian manifold with vanishing C-Bochner curvature tensor and $\operatorname{Tr} \operatorname{Ric}^{(m)}$ is constant for a positive integer $m$.

\footnotetext{
${ }^{*}$ Supported by TGRC-KOSEF.
} 


\section{Preliminaries}

Let $M$ be an $n$-dimensional Riemannian manifold. Throughout this paper, we assume that manifolds are connected and of class $C^{\infty}$. Denoting respectively by $g_{j i}, R_{k j i}{ }^{h}, R_{j i}=R_{r j i}{ }^{r}$ and $R$ the metric tensor, the curvature tensor, the Ricci tensor and the scalar curvature of $M$ in terms of local coordinates $\left\{x^{h}\right\}$, where Latin indices run over the range $\{1,2, \ldots, n\}$.

An $n(=2 l+1)$-dimensional Riemannian manifold is called a Sasakian manifold if there exists a unit Killing vector field $\xi^{h}$ satisfying

$$
\begin{cases}\eta_{i}=g_{i r} \xi^{r}, \quad \phi_{j i}=\nabla_{j} \eta_{i}, & \phi_{j i}+\phi_{i j}=0, \quad \phi_{r}^{h} \xi^{r}=0, \quad \phi_{j}^{r} \eta_{r}=0 \\ \phi_{i}{ }^{r} \phi_{r}{ }^{h}=-\delta_{i}{ }^{h}+\eta_{i} \xi^{h}, & \nabla_{k} \phi_{j i}=-g_{k j} \eta_{i}+g_{k i} \eta_{j}\end{cases}
$$

where $\nabla$ denotes the operator of the Riemannian covariant derivative.

It is well known that in a Sasakian manifold the following equations hold:

(2.2) $R_{j r} \xi^{r}=(n-1) \eta_{j}$

(2.3) $H_{j i}+H_{i j}=0$,

(2.4) $R_{j i}=R_{r s} \phi_{j}{ }^{r} \phi_{i}{ }^{s}+(n-1) \eta_{j} \eta_{i}$,

$(2.5) \nabla_{k} R_{j i}-\nabla_{j} R_{k i}=\left(\nabla_{t} R_{k r}\right) \phi_{j}{ }^{r} \phi_{i}{ }^{t}-\eta_{j}\left\{H_{k i}-(n-1) \phi_{k i}\right\}-2 \eta_{i}\left\{H_{k j}-(n-1) \phi_{k j}\right\}$,

(2.6) $\nabla_{k} R_{j i}-\left(\nabla_{k} R_{r s}\right) \phi_{j}{ }^{r} \phi_{i}{ }^{s}=-\eta_{i}\left\{H_{k j}-(n-1) \phi_{k j}\right\}-\eta_{j}\left\{H_{k i}-(n-1) \phi_{k i}\right\}$,

(2.7) $\xi^{r} \nabla_{r} R_{k j i}{ }^{h}=0$,

where we put $H_{j i}=\phi_{j}^{r} R_{r i}$.

We denote a tensor field $\operatorname{Ric}^{(m)}$ with components $R_{j i}^{(m)}$ and a function $R_{(m)}$ as follows:

$$
R_{j i}^{(m)}=R_{j i_{1}} R_{i_{2}}^{i_{1}} \cdots R_{i}^{i_{m-1}}, \quad R_{(m)}=\operatorname{Tr} \operatorname{Ric}^{(m)}=g^{j i} R_{j i}^{(m)} .
$$

Then, from (2.2) and (2.3), we get

$$
\begin{aligned}
& R_{j r}^{(m)} \xi^{r}=(n-1)^{m} \eta_{j}, \\
& R_{j r}^{(m)} \phi_{i}{ }^{r}+R_{i r}{ }^{(m)} \phi_{j}^{r}=0 .
\end{aligned}
$$

Also, we define the $\eta$-Eintein tensor $T_{j i}$ by

$$
T_{j i}=R_{j i}-\left(\frac{R}{n-1}-1\right) g_{j i}+\left(\frac{R}{n-1}-n\right) \eta_{j} \eta_{i} .
$$

If the $\eta$-Einstein tensor vanishes, then $M$ is called an $\eta$-Einstein manifold. From (2.2) and (2.3), we have

$$
\begin{aligned}
& \operatorname{Tr} T=0, \\
& T_{j r} \xi^{r}=0, \\
& T_{j r} \phi_{i}^{r}+T_{i r} \phi_{j}^{r}=0 .
\end{aligned}
$$


A Sasakian manifold $M$ is called a space of constant $\phi$-holomorphic sectional curvature $c$ if the curvature tensor of $M$ has the form:

$$
\begin{aligned}
R_{k j i}{ }^{h}= & \frac{c+3}{4}\left(g_{j i} \delta_{k}{ }^{h}-g_{k i} \delta_{j}{ }^{h}\right) \\
& +\frac{c-1}{4}\left(g_{k i} \eta_{j} \xi^{h}-g_{j i} \eta_{k} \xi^{h}+\eta_{k} \eta_{i} \delta_{j}{ }^{h}-\eta_{j} \eta_{i} \delta_{k}{ }^{h}-\phi_{k i} \phi_{j}{ }^{h}+\phi_{j i} \phi_{k}{ }^{h}-2 \phi_{k j} \phi_{i}{ }^{h}\right) .
\end{aligned}
$$

Matsumoto and Chūman ([10]) introduced the C-Bochner curvature tensor $B_{k j i}{ }^{h}$ defined by

$$
\begin{aligned}
& B_{k j i}{ }^{h}= R_{k j i}{ }^{h}+\frac{1}{n+3}( \\
&-H_{k i} \delta_{j}{ }^{h}-R_{j i} \delta_{k}{ }^{h}+g_{k i} R_{j}{ }^{h}-g_{j i} R_{k}{ }^{h}+H_{k i} \phi_{j}{ }^{h}+\phi_{k i} H_{j}{ }^{h}-\phi_{j i} H_{k}{ }^{h}+2 H_{k j} \phi_{i}{ }^{h}+2 \phi_{k j} H_{i}{ }^{h} \\
&\left.\quad-R_{k i} \eta_{j} \xi^{h}+R_{j i} \eta_{k} \xi^{h}-\eta_{k} \eta_{i} R_{j}{ }^{h}+\eta_{j} \eta_{i} R_{k}{ }^{h}\right) \\
&- \frac{k+n-1}{n+3}\left(\phi_{k i} \phi_{j}{ }^{h}-\phi_{j i} \phi_{k}{ }^{h}+2 \phi_{k j} \phi_{i}{ }^{h}\right) \\
&-\frac{k-4}{n+3}\left(g_{k i} \delta_{j}{ }^{h}-g_{j i} \delta_{k}{ }^{h}\right) \\
&+\frac{k}{n+3}\left(g_{k i} \eta_{j} \xi^{h}-g_{j i} \eta_{k} \xi^{h}+\eta_{k} \eta_{i} \delta_{j}{ }^{h}-\eta_{j} \eta_{i} \delta_{k}{ }^{h}\right)
\end{aligned}
$$

where $k=\frac{R+n-1}{n+1}$. It is well-known that if a Sasakian manifold with vanishing C-Bochner curvature tensor is an $\eta$-Einstein manifold, then it is a space of constant $\phi$-holomorphic sectional curvature.

\section{A Sasakian manifold with vanishing C-Bochner curvature tensor.}

Let $M^{n}(n \geq 5)$ be a Sasakain manifold with vanishing C-Bochner curvature tensor. By a straitforward computation, we can prove

$$
\begin{aligned}
\frac{n+3}{n-1} \nabla_{r} B_{k j i}{ }^{r}= & \nabla_{k} R_{j i}-\nabla_{j} R_{k i}-\eta_{k}\left\{H_{j i}-(n-1) \phi_{j i}\right\} \\
+ & \eta_{j}\left\{H_{k i}-(n-1) \phi_{k i}\right\}+2 \eta_{i}\left\{H_{k j}-(n-1) \phi_{k j}\right\} \\
+ & \frac{1}{2(n+1)}\left\{\left(g_{k i}-\eta_{k} \eta_{i}\right) \delta_{j}{ }^{r}-\left(g_{j i}-\eta_{j} \eta_{i}\right) \delta_{k}{ }^{r}\right. \\
& \left.+\phi_{k i} \phi_{j}{ }^{r}-\phi_{j i} \phi_{k}{ }^{r}+2 \phi_{k j} \phi_{i}{ }^{r}\right\} R_{r},
\end{aligned}
$$

where we put $R_{j}=\nabla_{j} R$.

By virtue of (2.1), (2.2), (2.5) - (2.7) and (3.1), we obtain

$$
\begin{aligned}
\nabla_{k} R_{j i}= & \left\{R_{k r}-(n-1) g_{k r}\right\}\left(\phi_{j}{ }^{r} \eta_{i}+\phi_{i}{ }^{r} \eta_{j}\right) \\
& +\frac{1}{2(n+1)}\left\{2 R_{k}\left(g_{j i}-\eta_{j} \eta_{i}\right)+R_{j}\left(g_{k i}-\eta_{k} \eta_{i}\right)\right. \\
& \left.\quad+R_{i}\left(g_{k j}-\eta_{k} \eta_{j}\right)-\phi_{k j} \phi_{i}{ }^{r} R_{r}-\phi_{k i} \phi_{j}{ }^{r} R_{r}\right\}
\end{aligned}
$$

and consequently from (2.7), we find

$$
(n+1)\left(\nabla_{k} R_{j i}\right) R^{j} R^{i}=2 \lambda^{2} R_{k},
$$


where we put $\lambda^{2}=R_{r} R^{r}$.

The following lemma is needed for the later use.

Lemma 3.1 Let $M^{n}(n \geq 5)$ be a Sasakian manifold with vanishing C-Bochner curvature tensor. Then $R_{j r}{ }^{(m)} R^{r}=0$ holds for a positive integer $m$ if and only if the scalar curvature $R$ is constant.

Proof. If $R_{j r}^{(m)} R^{r}=0$ holds, then we get $R_{j r}^{(2 m-2)} R^{r}=0$ which implies that $\left.R_{j r}^{(m-1)} R^{r}\right|^{2}=0$. Accordingly, we obtain $R_{j r}^{(m-1)} R^{r}=0$. By the inductive method, we get $R_{j r} R^{r}=0$. Operating $\nabla_{k}$ to this, we find $\left(\nabla_{k} R_{j r}\right) R^{j} R^{r}=0$. By means of (3.3), we see that the scalar curvature $R$ is constant. The converse is trivial.

For the sake of brevity, we shall define a function $\alpha(m)$ as follows:

$$
\alpha(m)=R_{j i}^{(m)} R^{j} R^{i} .
$$

Then, it is clear from (3.2) that

$(3.4) 2(n+1)\left(\nabla_{k} R_{j i}\right) R^{j}\left(R^{i r(m)} R_{r}\right)=\lambda^{2} R_{k r}^{(m)} R^{r}+3 \alpha(m) R_{k}$,

$(3.5) 2(n+1)\left(\nabla_{k} R_{j i}\right)\left(R^{j r(\ell)} R_{r}\right)\left(R^{i s(m)} R_{s}\right)=\alpha(\ell) R_{k r}^{(m)} R^{r}+\alpha(m) R_{k r}^{(\ell)} R^{r}+2 \alpha(\ell+m) R_{k}$,

where we have used (2.7), (2.8) and (2.9).

Operating $R^{j i(m)}$ to $(3.2)$ and owing to $(2.1),(2.7),(2.8)$ and (2.9), we find

$$
(n+1) \nabla_{k} R_{(m+1)}=(m+1)\left[2 R_{k r}^{(m)} R^{r}+\left\{R_{(m)}-(n-1)^{m}\right\} R_{k}\right] .
$$

Therefore, if the scalar curvature $R$ is constant, then $R_{(m)}$ is constant for any integer $m(\geq 2)$.

Now, we shall prove that the scalar curvature $R$ is constant if $R_{(m)}$ is constant for any fixed integer $m(\geq 2)$.

At first, suppose that $R_{(2 \ell+3)}(\ell=0,1,2, \ldots)$ is constant. Then, from (3.6), we can get

$$
2 R_{k r}^{(2 \ell+2)} R^{r}+\left\{R_{(2 \ell+2)}-(n-1)^{2 \ell+2}\right\} R_{k}=0,
$$

which yields that $2 \alpha(2 \ell+2)+\lambda^{2}\left\{R_{(2 \ell+2)}-(n-1)^{2 \ell+2}\right\}=0$, that is,

$$
2\left|R_{j r}^{(\ell+1)} R^{r}\right|^{2}+\lambda^{2}\left|R_{j i}^{(\ell+1)}-(n-1)^{\ell+1} \eta_{j} \eta_{i}\right|^{2}=0 .
$$

Thus, from Lemma 3.1, the scalar curvature $R$ is constant.

In the next place, we shall consider when $R_{(2 \ell+2)}(\ell=0,1,2, \ldots)$ is constant. From (3.6), we have

$$
2 R_{j r}^{(2 \ell+1)} R^{r}+\left\{R_{(2 \ell+1)}-(n-1)^{2 \ell+1}\right\} R_{j}=0 .
$$

Operating $\nabla_{k}$ to this and owing to (3.7), we get

$$
2\left(\nabla_{k} R_{j r}^{(2 \ell+1)}\right) R^{j} R^{r}+\lambda^{2} \nabla_{k} R_{(2 \ell+1)}=0 .
$$

From (3.3) and (3.8), we find the scalar curvature $R$ is constant if $\ell=0$. Because of (3.4), (3.5) and (3.6), equation (3.8) is rewritten as follows:

$$
\begin{aligned}
& 4(\ell+1) \lambda^{2} R_{k r}^{(2 \ell)} R^{r}+2 \sum_{i=1}^{2 \ell-1} \alpha(i) R_{k r}^{(2 \ell-i)} R^{r} \\
& +4(\ell+1) \alpha(2 \ell) R_{k}+(2 \ell+1) \lambda^{2}\left|R_{j i}^{(\ell)}-(n-1)^{\ell} \eta_{j} \eta_{i}\right|^{2} R_{k}=0 .
\end{aligned}
$$


By virtue of (3.9) and Lemma 3.1, it is clear that the scalar curvature $R$ is constant if $\ell=1$.

On the other hand, we have

$$
\begin{aligned}
& \lambda^{6} \alpha(2 \ell)+2 \lambda^{4} \alpha(s) \alpha(2 \ell-s)+\lambda^{4} \alpha(2 s) \alpha(2 \ell-2 s) \\
& =\lambda^{2}\left|\lambda^{2} R_{j r}^{(\ell)} R^{r}+\alpha(s) R_{j r}^{(\ell-s)} R^{r}\right|^{2}+\alpha(2 \ell-2 s)\left|\lambda^{2} R_{j r}^{(s)} R^{r}-\alpha(s) R_{j}\right|^{2} .
\end{aligned}
$$

Because of (3.9) and (3.10), it is to see that the following equations hold: if $\ell=2,6,10, \ldots$,

$$
\begin{aligned}
& (7 \ell+8) \lambda^{6} \alpha(2 \ell)+(2 \ell+1) \lambda^{8}\left|R_{j i}^{(\ell)}-(n-1)^{\ell} \eta_{j} \eta_{i}\right|^{2} \\
& +4 \lambda^{4} \sum_{i=1}^{(\ell-2) / 4} \alpha(4 i) \alpha(2 \ell-4 i) \\
& +2 \lambda^{2} \sum_{i=1}^{\ell / 2}\left|\lambda^{2} R_{j s}^{(\ell)} R^{s}+\alpha(2 i-1) R_{j s}^{(\ell-2 i+1)} R^{s}\right|^{2} \\
& +2 \sum_{i=1}^{\ell / 2} \alpha(2 \ell-4 i+2)\left|\lambda^{2} R_{j s}^{(2 i-1)} R^{s}-\alpha(2 i-1) R_{j}\right|^{2}=0,
\end{aligned}
$$

if $\ell=4,8,12, \ldots$,

$$
\begin{aligned}
& (7 \ell+8) \lambda^{6} \alpha(2 \ell)+(2 \ell+1) \lambda^{8}\left|R_{j i}^{(\ell)}-(n-1)^{\ell} \eta_{j} \eta_{i}\right|^{2} \\
& +4 \lambda^{4} \sum_{i=1}^{(\ell-4) / 4} \alpha(4 i) \alpha(2 \ell-4 i)+2 \lambda^{4} \alpha(\ell)^{2} \\
& +2 \lambda^{2} \sum_{i=1}^{\ell / 2}\left|\lambda^{2} R_{j s}^{(\ell)} R^{s}+\alpha(2 i-1) R_{j s}^{(\ell-2 i+1)} R^{s}\right|^{2} \\
& +2 \sum_{i=1}^{\ell / 2} \alpha(2 \ell-4 i+2)\left|\lambda^{2} R_{j s}^{(2 i-1)} R^{s}-\alpha(2 i-1) R_{j}\right|^{2}=0
\end{aligned}
$$

and if $\ell=3,5,7, \ldots$,

$$
\begin{aligned}
& (7 \ell+9) \lambda^{6} \alpha(2 \ell)+(2 \ell+1) \lambda^{8}\left|R_{j i}^{(\ell)}-(n-1)^{\ell} \eta_{j} \eta_{i}\right|^{2} \\
& +2 \lambda^{4} \sum_{i=1}^{(\ell-1) / 2} \alpha(2 i) \alpha(2 \ell-2 i)+2 \lambda^{4} \alpha(\ell)^{2} \\
& +2 \lambda^{2} \sum_{i=1}^{(\ell-1) / 2}\left|\lambda^{2} R_{j s}^{(\ell)} R^{s}+\alpha(2 i-1) R_{j s}^{(\ell-2 i+1)} R^{s}\right|^{2} \\
& +2 \sum_{i=1}^{(\ell-1) / 2} \alpha(2 \ell-4 i+2)\left|\lambda^{2} R_{j s}^{(2 i-1)} R^{s}-\alpha(2 i-1) R_{j}\right|^{2}=0 .
\end{aligned}
$$

Thus we find from Lemma 3.1 that the scalar curvature $R$ is constant if $R_{(2 \ell+2)}(\ell=$ $2,3,4, \ldots)$ is constant. Hence, we have

TheOREM 3.2 Let $M^{n}(n \geq 5)$ be a Sasakian manifold with vanishing C-Bochner curvature tensor. Then the scalar curvature $R$ is constant if and only if $\operatorname{Tr} \operatorname{Ric}^{(m)}$ is constant for an integer $m(\geq 2)$. 
REMark. In the proof of Theorem 3.2, we use only equation (3.1). Thus Theorem 3.2 is valid for the parallel C-Bochner curvature tensor.

Also, we have from Theorems B and 3.2

Theorem 3.3 Let $M^{n}(n \geq 5)$ be a Sasakian manifold whose $C$-Bochner curvature tensor vanishes. If the Ricci tensor is positive semi-definite and $\operatorname{Tr}_{\mathrm{Ric}}^{(m)}$ is constant for a positive integer $m$, then $M$ is a space of constant $\phi$-holomorphic sectional curvature.

Furthermore, it is easy to see from the proof of Theorem C and Theorem 3.2 that the following theorem hold:

Theorem 3.4 Let $M^{n}(n \geq 5)$ be a Sasakian manifold with vanishing C-Bochner curvature tensor. If $\operatorname{Tr} \operatorname{Ric}^{(m)}$ is constant for a positive integer $m$ and the length of the $\eta$-Einstein tensor is less than $\frac{\sqrt{2}(R-n+1)}{\sqrt{(n-1)(n-3)}}$, then $M$ is a space of constant $\phi$-holomorphic sectional curvature.

\section{The smallest Ricci curvature.}

Let $M$ be an $n(\geq 5)$-dimensional Sasakian manifold with vanishing C-Bochner curvature tensor. Suppose that $R_{(m)}$ is constant for any positive integer $m$. By Theorem 3.2, equation (3.2) is reduced to

$$
\nabla_{k} R_{j i}=\left\{R_{k r}-(n-1) g_{k r}\right\}\left(\phi_{j}{ }^{r} \eta_{i}+\phi_{i}{ }^{r} \eta_{j}\right),
$$

which implies $\nabla_{k} R_{j i}+\nabla_{j} R_{i k}+\nabla_{i} R_{k j}=0$, namely, the Ricci tensor is cyclic parallel. Therefore, using the Ricci formula, we find

$$
\nabla^{k} \nabla_{k} R_{j i}=2\left(R_{r j i s} R^{r s}-R_{j i}^{(2)}\right) .
$$

Applying $\nabla^{k}$ to (4.1) and owing to (2.1) and (2.2), we get

$$
\nabla^{k} \nabla_{k} R_{j i}=-2\left[R_{j i}-(n-1) g_{j i}-\{R-n(n-1)\} \eta_{j} \eta_{i}\right] .
$$

On the other hand, by virtue of (2.1) - (2.4) and (2.14), it is clear that the following equation holds:

$$
\begin{aligned}
(n+3) R_{r j i s} R^{r s}= & 4 R_{j i}^{(2)}-(4 n-R+2 k) R_{j i}+\left\{R_{(2)}-(k-4) R+(n-1) k\right\} g_{j i} \\
& -\left\{R_{(2)}+(n-1)^{2}-(n-1) k-k R\right\} \eta_{j} \eta_{i} .
\end{aligned}
$$

From the last three equations, we have

$$
R_{j i}^{(2)}=\beta R_{j i}+\gamma g_{j i}+\left\{(n-1)^{2}-(n-1) \beta-\gamma\right\} \eta_{j} \eta_{i},
$$

where constants $\beta$ and $\gamma$ are given by

$$
\begin{aligned}
& (n+1) \beta=R-3 n-5 \\
& (n-1) \gamma=R_{(2)}-\frac{1}{n+1} R^{2}+4 R-\frac{n-1}{n+1}\left(n^{2}+3 n+4\right) .
\end{aligned}
$$

Thus, equation (4.2) tells us that $M$ has at most three constant Ricci curvatures $n-1$, $x_{1}$ and $x_{2}$, where we have put

$$
x_{1}=\frac{1}{2}(\beta-\sqrt{D}), \quad x_{2}=\frac{1}{2}(\beta+\sqrt{D}), \quad D=\beta^{2}+4 \gamma(\geq 0),
$$


moreover, the multiplicities of $x_{1}$ and $x_{2}$ denote by $s$ and $n-1-s$, respectively. Therefore we have (cf. [7])

Lemma 4.1 Let $M^{n}(n \geq 5)$ be a Sasakian manifold with vanishing C-Bochner curvature tensor such that $\operatorname{Tr} \operatorname{Ric}^{(m)}$ is constant for a positive integer $m$. Then $M$ has at most three constant Ricci curvatures.

Now, we shall prove the following theorem.

TheOREM 4.2 Let $M^{n}(n \geq 5)$ be a Sasakian manifold with vanishing $C$-Bochner curvature tensor such that $\operatorname{Tr} \operatorname{Ric}^{(m)}$ is constant for a positive integer $m$. If the smallest Ricci curvature is greater than or equal to -2 , then $M$ is a space of constant $\phi$-holomorphic sectional curvature -3 .

Proof. By means of (4.3), (4.5) and Lemma 4.1, we find

$$
R+n-1=\frac{n+1}{n+3}(n-1-2 s) \sqrt{D} .
$$

Because of (4.3), (4.4) and (4.6), we have

$\frac{n-1}{4}\left\{1-\left(\frac{n-1-2 s}{n+3}\right)^{2}\right\} D=R_{(2)}-\frac{1}{n+1}\left\{R^{2}-2(n+3) R+(n-1)^{2}(n+2)\right\}$,

which yields that

$$
(n+1) R_{(2)} \geq R^{2}-2(n+3) R+(n-1)^{2}(n+2) .
$$

Let $x_{1}$ be the smallest Ricci curvature. Then, by virtue of (4.5), we obtain $\gamma \leq 2 \beta+4$ which means from (4.4) that

$$
(n+1) R_{(2)} \leq R^{2}-2(n+3) R+(n-1)^{2}(n+2) .
$$

Combining this with (4.7), we get $D$ vanishes identically, which implies that equation (4.6) gives $R=-n+1$. We find $\left|R_{j i}+2 g_{j i}-(n+1) \eta_{j} \eta_{i}\right|^{2}=0$ which yields that $M$ is an $\eta$ Einstein manifold. Thus, it is easy to see from (2.14) that $M$ is of constant $\phi$-holomorphic sectional curvature -3 .

REMARK. In [10], this theorem was proved under the condition that $M$ is compact.

\section{REFERENCES}

[1] S. Bochner, Curvatures and Betti numbers, II, Annals of Math., 50 (1949), 77-93.

[2] W. M. Boоthby And H. C. Wang, On contact manifolds, Annals of Math., 68 (1958), 721-734.

[3] I. HasegaWA, Sasakian manifolds with $\eta$-parallel contact Bochner curvature tensor, $J$. Hokka- ido Univ. Ed. Sect. II A. 29 (1979), 1-5.

[4] I. Hasegawa AND T.NAKAne, On Sasakian manifolds with vanishing contact Bochner curvature tensor, Hokkaido Math. J. 9 (1980), 184-189.

[5] ( On Sasakian manifolds with vanishing contact Bochner curvature tensor II, Hokkaido Math. J. 11 (1982), 44-51. 
[6] U-H. Ki And B. H. KIM, Manifolds with Kaehler-Bochner metric, Kyungpook Math. J. 32 (1992), 285-290.

[7] U-H. Ki AND H. S. Kim, Sasakian manifolds whose C-Bochner curvature tensor vanishes, Tensor, N. S. 49 (1990), 32-39.

[8] Y. Kubo, Kaehlerian manifolds with vanishing Bochner curvature tensor, Kōdai Math. Sem. Rep. 28 (1976), 85-89.

[9] M. Matsumoto, On Kählerian space with parallel or vanishing Bochner curvature tensor, Tensor N. S. 20 (1969), 25-28.

[10] M. Matsumoto and G. Chūman, On the C-Bochner curvature tensor, TRU Math. $\mathbf{5}$ (1969), 21-30.

[11] M. Matsumoto And S. Tanno, Kählerian spaces with parallel or vanishing Bochner curvature tensor, Tensor N. S. 27 (1973), 291-294.

[12] J. S. PAK, A note on Sasakian manifolds with vanishing C-Bochner curvature tensor, Kōdai Math. Sem. Rep. 28 (1976), 19-27.

[13] S. Tachibana, On the Bochner curvature tensor, Nat. Scie. Rep. Ochanomizu Univ. 18 (1967), 15-19.

[14] Y. Tashiro and S. Tachibana, On Fubinian and C-Fubinian manifolds, Kōdai Math. Sem. Rep. 15 (1963), 176-183.

[15] K. Yano And S. Bochner, Curvature and Betti numbers, Annals of Math. Stud. 32 1953.

[16] K. Yano And S. Ishinara, Kaehlerian manifolds with constant scalar curvature whose Bochner curvature tensor vanishes, Hokkaido Math. J. 3 (1974), 294-304.

\author{
YEUNGNAM UNIVERSITY \\ KYUNGSAN 712-749 \\ KOREA \\ TOPOLOGY AND GEOMETRY RESEARCH CENTER \\ KYUNGPOOK NATIONAL UNIVERSITY \\ TAEGU 702-701 \\ KOREA \\ DEPARTMENT OF MATHEMATICS \\ FACULTY OF ENGINEERING \\ SHINSHU UNIVERSITY \\ NAGANO, 380 \\ JAPAN
}

